\title{
Front Matter: Volume 7990
}

, "Front Matter: Volume 7990," Proc. SPIE 7990, Optical Sensors and Biophotonics II, 799001 (23 February 2011); doi: 10.1117/12.890180

SPIE Event: Asia Communications and Photonics Conference and Exhibition, 2010, Shanghai, Shanghai, China 


\title{
PROCEEDINGS OF SPIE
}

\section{Optical Sensors and Biophotonics II}

\author{
Qingming Luo \\ Frank Chuang \\ Stefan Haacke \\ Chih-Chung Yang \\ Editors
}

\section{8-12 December 2010 \\ Shanghai, China}

\author{
Cosponsored by \\ IEEE Photonics Society • Optical Society of America • SPIE • Chinese Optical Society \\ China Institute of Communications \\ Local Organizing Committee \\ Fudan University - Pudong Optoelectronics Industry Association of Shanghai \\ Shanghai Jiao Tong University - Shanghai Institute of Optics and Fine Mechanics \\ Alcatel-Lucent Shanghai Bell \\ Best Student Paper Sponsor \\ Thorlabs, Inc. \\ Published by \\ SPIE • Optical Society of America • IEEE Photonics Society
}

Volume 7990 
The papers included in this volume were part of the technical conference cited on the cover and title page. Papers were selected and subject to review by the editors and conference program committee. Some conference presentations may not be available for publication. The papers published in these proceedings reflect the work and thoughts of the authors and are published herein as submitted. The publisher is not responsible for the validity of the information or for any outcomes resulting from reliance thereon.

Please use the following format to cite material from this book:

Author(s), "Title of Paper," in Optical Sensors and Biophotonics II, edited by Qingming Luo, Frank Chuang, Stefan Haacke, Chih-Chung Yang, Proceedings of SPIE-OSA-IEEE Asia Communications and Photonics, Vol. 7990 (SPIE, Bellingham, WA, 2011) Article CID Number.

ISSN 0277-786X

ISBN 9780819485588

Published by

SPIE

P.O. Box 10, Bellingham, Washington $98227-0010$ USA

Telephone +1 3606763290 (Pacific Time) · Fax +1 3606471445

SPIE.org

Optical Society of America

2010 Massachusetts Ave., N.W., Washington, D.C., 20036 USA

Telephone +1 2022238130 (Eastern Time) · Fax +1 2022231096

OSA.org

IEEE Photonics Society

445 Hoes Lane, Piscataway, New Jersey, 08855 USA

Telephone +1 7325628434 (Eastern Time) · Fax +1 7325628434

IEEE.org

Copyright (c) 201 1, Society of Photo-Optical Instrumentation Engineers, Optical Society of America, and IEEE Photonics Society.

Copying of material in this book for internal or personal use, or for the internal or personal use of specific clients, beyond the fair use provisions granted by the U.S. Copyright Law is authorized by SPIE subject to payment of copying fees. The Transactional Reporting Service base fee for this volume is $\$ 18.00$ per article (or portion thereof), which should be paid directly to the Copyright Clearance Center (CCC), 222 Rosewood Drive, Danvers, MA 01923. Payment may also be made electronically through CCC Online at copyright.com. Other copying for republication, resale, advertising or promotion, or any form of systematic or multiple reproduction of any material in this book is prohibited except with permission in writing from the publisher. The CCC fee code is $0277-786 \mathrm{X} / 11 / \$ 18.00$.

Printed in the United States of America.

Publication of record for individual papers is online in the SPIE Digital Library.

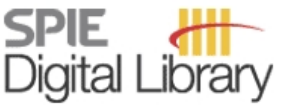

SPIEDigitalLibrary.org

Paper Numbering: Proceedings of SPIE follow an e-First publication model, with papers published first online and then in print and on CD-ROM. Papers are published as they are submitted and meet publication criteria. A unique, consistent, permanent citation identifier (CID) number is assigned to each article at the time of the first publication. Utilization of CIDs allows articles to be fully citable as soon they are published online, and connects the same identifier to all online, print, and electronic versions of the publication. SPIE uses a six-digit CID article numbering system in which:

- The first four digits correspond to the SPIE volume number.

- The last two digits indicate publication order within the volume using a Base 36 numbering system employing both numerals and letters. These two-number sets start with 00, 01, 02, 03, 04, 05, 06, 07, 08, 09, 0A, OB ... OZ, followed by 10-1Z, 20-2Z, etc.

The CID number appears on each page of the manuscript. The complete citation is used on the first page, and an abbreviated version on subsequent pages. Numbers in the index correspond to the last two digits of the six-digit CID number. 


\section{Contents}

vii Symposium Committees
ix Conference Committee

\section{BIOPHOTONICS FOR CLINICAL APPLICATION}

799002 Clinical translation of ultraviolet autofluorescence microscopy towards endomicroscopy for early detection of cancer [7990-31]

B. Lin, S. Urayama, R. M. G. Saroufeem, D. L. Matthews, Univ. of California, Davis (United States); S. G. Demos, Univ. of California, Davis (United States) and Lawrence Livermore National Lab. (United States)

\section{OPTICAL SENSORS}

799003 Multi-longitudinal mode fiber laser sensor combining the fiber Bragg grating reflector [7990-30]

S. Liu, Nanjing Univ. (China) and Heilongjiang Univ. (China); L. Gao, Z. Yin, L. Zhang, X. Chen, J. Cheng, Nanjing Univ. (China)

$799004 \quad$ A biconical taper multi-mode fiber SERS sensor [7990-23]

H. Bai, Z. Chen, N. Chen, Q. Guo, K. Zhang, F. Pang, T. Wang, Shanghai Univ. (China)

799005 Coupling characteristics between slot plasmonic mode and dielectric waveguide mode [7990-07]

S. Hu, F. Liu, R. Wan, Y. Huang, Tsinghua Univ. (China)

\section{BEST STUDENT PAPER COMPETITION}

799006 Novel periodic microstructures fabricated by multi-exposure two-beam interference lithography [7990-18]

Y. Bai, A. P. Zhang, Zhejiang Univ. (China)

799007 Simultaneous measurement of strain and temperature using a high-birefringence fiber loop mirror and an erbium-doped fiber [7990-08]

J. Shi, S. Xiao, H. Chen, M. Zhu, M. Bi, Shanghai Jiao Tong Univ. (China)

799008 Hollow-core Bragg fiber and its application in trace gas sensing [7990-16]

L. Shi, W. Zhang, J. Jin, Y. Huang, J. Peng, Tsinghua Univ. (China)

799009 Liquid lens: advances in adaptive optics [7990-15]

S. P. Casey, Shanghai Jiao Tong Univ. (China) 
7990 OA Multimodal endoscopic imaging and Raman spectroscopy for improving in vivo diagnosis of gastric malignancies during clinical gastroscopy (Invited Paper) [7990-04]

M. S. Bergholt, W. Zheng, K. Lin, National Univ. of Singapore (Singapore); K. Y. Ho, K. G. Yeoh, M. Teh, J. B. Y. So, National Univ. of Singapore (Singapore) and National Univ. Hospital System (Singapore); Z. Huang, National Univ. of Singapore (Singapore)

\section{POSTER SESSION}

7990 OB LED spectrum measurement via low cost spectrum sensor on-a-chip [7990-14] C.-C. Chang, C.-C. Chen, N.-T. Lin, National Taipei Univ. of Technology (Taiwan, China); U. Kurokawa, B. I Choi, nanoLambda Inc. (United States)

7990 OC Experimental study on humidity sensing using a FBG sensor with polyimide coating [7990-03] F. Ding, L. Wang, N. Fang, Z. Huang, Shanghai Univ. (China)

7990 OD Temperature stability of high Ge-doped fibre Bragg grating [7990-27]

J. Guo, F. Tu, H. Wei, T. Deng, W. Tong, Yangtze Optical Fibre and Cable Co., Ltd. (China)

7990 OE Laser inspection for surface acoustic wave performance in SAW device [7990-32]

J. O. Park, W. K. Jang, Hanseo Univ. (Korea, Republic of)

7990 OF Theoretical investigation on Raman fiber amplifiers [7990-26]

S. Li, H. Gong, Y. Meng, Y. Tu, China Jiliang Univ. (China)

7990 0G Relative humidity sensor based on photonic crystal fiber with tapered and filled in polymer [7990-12]

T. Li, C.-L. Zhao, X. Dong, W. Qian, Y. Jin, S. Jin, China Jiliang Univ. (China)

$7990 \mathrm{OH}$ Signal processing of Sagnac fiber interferometer used as distributed sensor with wavelets [7990-24]

Y. Liu, J. Jia, P. Tao, G. Yin, Z. Tan, W. Ren, S. Jian, Beijing Jiaotong Univ. (China)

7990 OI MOEMS gyroscope based on acoustooptic mode coupling [7990-28]

S. LU, S. Chen, Y. Zhao, Beijing Institute of Technology (China)

7990 0J Plane displacement measurement of rigid body by laser speckle [7990-17]

C. Zhong, China Jiliang Univ. (China) and South-Central Univ. for Nationalities (China);

C. Shen, China Jiliang Univ. (China); Z. Lin, South-Central Univ. for Nationalities (China); K. Li, China Jiliang Univ. (China)

7990 OK Characteristics of refractive index sensor based on adjusting gap fiber Bragg grating [7990-01]

C. Shen, China Jiliang Univ. (China) and Zhejiang Univ. (China); C. Zhong, China Jiliang Univ. (China)

$7990 \mathrm{OL}$ The Influence of linear birefringence on the polarization properties of uniform fiber gratings [7990-09]

Y. SU, B. Zhang, Y. Li, The PLA Univ. of Science and Technology (China) 
7990 OM A novel temperature-insensitive package for fiber Bragg grating [7990-20] Y. Tu, H. Gong, S. Li, Y. Jin, China Jiliang Univ. (China)

7990 ON Distributed optical fiber temperature sensor applied in underground coal gasification system [7990-02]

J. Wang, C. Hu, Z. Zhang, H. Gong, Y. Jin, C. Shen, China Jiliang Univ. (China)

799000 Simultaneous measurements of vibration, temperature, and humidity using a SOA-based fiber Bragg grating laser [7990-22]

L. Wang, N. Fang, F. Ding, Z. Huang, Shanghai Univ. (China)

7990 OP Fiber optic intrinsic Fabry-Perot temperature sensor fabricated by femtosecond lasers [7990-21]

W. Wang, Shanghai Univ. (China) and Hebei United Univ. (China); F. Pang, N. Chen,

X. Zhang, L. Lan, D. Ding, T. Wang, Shanghai Univ. (China)

$79900 Q \quad$ Simultaneous measurement of displacement and temperature with a single singlemode-multimode-singlemode (SMS) fiber structure [7990-06]

Q. Wu, Y. Semenova, P. Wang, A. M. Hatta, G. Farrell, Dublin Institute of Technology (Ireland)

7990 OR A luminescent temperature sensor based on a tapered optical fiber coated with quantum dots [7990-25]

F. Zhang, Shanghai Univ. (China) and Shandong Univ. (China); B. Wang, F. Pang, T. Wang, Shanghai Univ. (China)

7990 OS The curvature measurement of Sagnac loop based on PMF [7990-10]

Y. Zhao, Y. Jin, H. Gong, J. Wang, China Jiliang Univ. (China)

7990 OT Analyte-filled fiber core assisted surface plasmon sensing [7990-29]

Y. Zhang, C. Zhou, L. Xia, H. Liu, D. Liu, Huazhong Univ. of Science \& Technology (China)

7990 OU High sensitivity dual microring sensor based on intensity detection [7990-33]

H. Yi, Peking Univ. (China); D. S. Citrin, Georgia Institute of Technology (United States) and Georgia Tech, CNRS (France); Z. Zhou, Peking Univ. (China) and Georgia Institute of Technology (United States)

7990 OV Silicon-nanowire-based optical sensor with high sensitivity and large measurement range by using Mach-Zehnder interferometer-coupled microring [7990-19]

J. Wang, S. He, D. Dai, Zhejiang Univ. (China)

\section{POST-DEADLINE PAPERS}

7990 OW Radiographic, microcomputer tomography, and optical coherence tomography investigations of ceramic interfaces [7990-38]

C. Sinescu, M. L. Negrutiu, Victor Babes Univ. of Medicine and Pharmacy, Timisoara (Romania); C. Ionita, Univ. at Buffalo (United States); F. Topala, E. Petrescu, R. Rominu, D. M. Pop, Victor Babes Univ. of Medicine and Pharmacy, Timisoara (Romania);

L. Marsavina, R. Negru, Politehnica Univ. Timisoara (Romania); A. Bradu, Univ. of Kent (United Kingdom); M. Rominu, Victor Babes Univ. of Medicine and Pharmacy, Timisoara (Romania);

A. G. Podoleanu, Univ. of Kent (United Kingdom) 
7990 OX Research on sensor design for internet of things and laser manufacturing [7990-37]

T. Wang, J. Yao, L. Guo, Y. Zhang, Hebei Univ. of Technology (China)

7990 OY Non-invasive imagistic investigation of metal-ceramic crowns [7990-39]

E. Petrescu, C. Sinescu, M. L. Negrutiu, R. Rominu, D. M. Pop, M. Rominu, Victor Babes Univ. of Medicine and Pharmacy, Timisoara (Romania)

$79900 Z$ The effect of different chemical agents on human enamel: an atomic force and scanning electron microscopy study [7990-40]

R. O. Rominu, M. Rominu, M. L. Negrutiu, C. Sinescu, D. Pop, E. Petrescu, Victor Babes Univ. of Medicine and Pharmacy, Timisoara (Romania)

Author Index 


\title{
Symposium Committees
}

\author{
General Chairs
}

Weishing Hu, Shanghai Jiao Tong University (China)

Ming-Jun Li, Corning, Inc. (United States)

Dennis L. Matthews, University of California, Davis (United States)

Technical Program Chairs

Dominique Chiaroni, Alcatel-Lucent (France)

Jian-Jun He, Zhejiang University (China)

Ken-ichi Kitayama, Osaka University (Japan)

Xingde Li, Johns Hopkins University (United States)

Local Organizing Committee

Nan Chi (Chair), Fudan University (China)

Chansai Cheng, Pudong Optoelectronics Industry Association of

Shanghai (China)

Meiyu Cao, Pudong Optoelectronics Industry Association of Shanghai (China)

Yaohui Jin, Shanghai Jiao Tong University (China)

Ronghui Qu, Shanghai Institute of Optics and Fine Mechanics (China)

Feng Huang, Alcatel-Lucent Shanghai Bell (China)

Organizing Committee

Lirong Zheng, Fudan University (China)

Liangyao Chen, Fudan University (China)

Yiwei Shi, Fudan University (China)

Bo Hu, Fudan University (China)

Rongjun Zhang, Fudan University (China)

Zhengcai Bian, Fudan University (China)

Jianjun Yin, Fudan University (China)

Xinyue Guo, Fudan University (China)

Xiaolu Wan, Fudan University (China)

General Secretariat

Yufeng Shao, Fudan University (China) 
Steering Committee Chairs

Kwok-Wai Cheung, Chinese University of Hong Kong (Hong Kong, China)

Thomas Koch, Lehigh University (United States)

John Zyskind, Mintera Corporation (United States)

Steering Committee Members

Connie Chang-Hasnain, University of California, Berkeley (United States)

Chongcheng Fan, Tsinghua University (China)

Sailing He, Zheijang University (China)

Peter Kaiser, Telcordia Technologies (retired) (United States)

Thomas Koch, Lehigh University (United States)

Ming-Jun Li, Corning, Inc. (United States)

Tingye Li, AT\&T Laboratories (retired) (United States)

Mao Qian, WRI-Fiberhome (China)

Xiaomin Ren, Beijing University of Posts and Telecommunications (China)

Bingkun Zhou, Tsinghua University (China) 


\title{
Conference Committee
}

\author{
Conference Chair
}

Qingming Luo, Huazhong University of Science and Technology (China)

\section{Conference Cochairs}

Frank Chuang, Lawrence Livermore National Laboratory (United States)

Stefan Haacke, Institut de Physique et Chimie des Matériaux de Strasbourg (France)

Chih-Chung Yang, National Taiwan University (Taiwan, China)

\section{Program Committee}

Yoshiaki Yasuno, University of Tsukuba (Japan)

Lihong Wang, Washington University in St. Lovis (United States)

Min Gu, Swinburne University of Technology (Australia)

Xingde Li, Johns Hopkins University (United States)

Claude Boccara, École Supérieure de Physique et de Chimie Industrielles de la Ville de Paris (France)

Jin U. Kang, Johns Hopkins University (United States)

Haishan Zeng, British Columbia Cancer Research Center (Canada)

Yu Chen, University of Maryland (United States)

Da Xing, South China Normal University (China)

Zhiwei Huang, National University of Singapore (Singapore)

Chii-Wann Lin, National Taiwan University (Taiwan, China)

J.-L. Martin, Optics Graduate School \& Laboratory for Optics and Bioscience (France)

Chi-Kuang Sun, National Taiwan University (Taiwan, China)

Kexin Xu, Tianjin University (China)

Shean-Jen Chen, National Cheng Kung University (Taiwan, China)

Pengcheng Li, Huazhong University of Science and Technology (China)

\section{Session Chairs}

ThE Biophotonics for Clinical Application

Qingming Luo, Huazhong University of Science and Technology

(China) 
ThJ Optical Sensors

Yoshiaki Yasuno, University of Tsukuba (Japan)

FE Cellular and Molecular Optical Imaging

Dennis L. Matthews, University of California, Davis (United States)

FL Optical Coherence Tomography I

Chih-Chung Yang, National Taiwan University (Taiwan, China)

FS Best Student Paper Competition

Nanguang Chen, National University of Singapore (Singapore)

SD Raman/Fluorescence Spectroscopy and Imaging

Zhongping Chen, University of California, Irvine (United States)

SJ Nonlinear Optical Imaging

Stefan Haacke, Institut de Physique et Chimie des Matériaux de Strasbourg (France)

SuD Optical Coherence Tomography II and Photoacoustic Imaging Jin U. Kang, Johns Hopkins University (United States)

SuJ Micro/Nano-Biophotonics

Da Xing, South China Normal University (China)

SuN Laser Speckle Imaging

Shean-Jen Chen, National Cheng Kung University (Taiwan, China) 